\title{
Optical realization of universal quantum cloning
}

\author{
Yun-Feng Huang, Wan-Li Li, Chuan-Feng Li*, Yong-Sheng Zhang, Yun-Kun Jiang, Guang-Can Guo ${ }^{\dagger}$ \\ Laboratory of Quantum Communication and Quantum Computation and Department \\ of Physics, University of Science and Technology of China, \\ Hefei, 230026, P. R. China
}

\begin{abstract}
Beyond the no-cloning theorem, the universal symmetric quantum cloning machine was first addressed by Bužek and Hillery. Here, we realized the one-to-two qubits Bužek-Hillery cloning machine with linear optical devices. This method relies on the representation of several qubits by a single photon. We showed that, the fidelities between the two output qubits and the original qubit are both $\frac{5}{6}$ (which proved to be the optimal fidelity of one-to-two qubits universal cloner) for arbitrary input pure states.

PACS number(s): 03.67.-a, 03.65.Bz
\end{abstract}

\section{INTRODUCTION}

A deterministic and perfect quantum cloner for arbitrary quantum states is forbidden by the quantum nocloning theorem [1]. As an extension, Yuen and D'Ariano [2] showed that a violation of unitarity makes the cloning of two nonorthogonal states also impossible. Then the case of mixed states is considered and it is shown that two noncommuting mixed states cannot be broadcast, even when the state need only be reproduced marginally on the separate systems. For further consideration, Koashi and Imoto [3] generalized the standard no-cloning theorem to the entangled states. Their research showed to what extent the correlation to other systems can be read out from a subsystem without altering its marginal density operators.

After having considered about so many restrictions posed on cloning quantum states by quantum mechanics, people began to think about how to do our best in quantum states cloning under all these restrictions, that is, the inaccurate cloning of quantum states. These approached may be divided into two main categories: universal and state-dependent.

State-dependent quantum cloning machines are designed to clone states belonging to a finite set. It can be divided into three subcategories: deterministic [4], probabilistic exact [5] and hybrid [6].

On the other hand, universal quantum cloning machines deterministically generates approximate copies of an unknown quantum state. The word "universal" means

*Electronic Address: cfli@ustc.edu.cn

†Electronic Address: gcguo@ustc.edu.cn that this kind of machines works equally well for any input states, i.e., state-independent. This category can again be divided into two subcategories: symmetric and asymmetric.

Asymmetric universal quantum cloning machines which introduced by Cerf and Bužek et al [7,8] produce two non-identical imperfect copies of a single state of $N$-dimensional quantum system. Symmetric universal quantum cloning machines, first designed by Bužek and Hillery [4], works in a different way: act on any unknown quantum state and produce identical approximate copies equally well. This kind of Bužek-Hillery cloning machine has been optimized and generalized [9, 10].

Quantum cloning may be used to improve the performance of some quantum computation tasks 11] and may be heleful in understanding quantum information theory and processing quantum imformation, and might be helpful in eavesdropping versus quantum cryptography [12]. And we can improve the performance of the measurements on observables by performing measurements on clones of the original quantum system [13].

In the present paper, we realized the one-to-two qubits Bužek-Hillery cloning machine with linear optical devices. This method relies on the representation of several qubits by a single photon. We showed that, the fidelities between the two output qubits and the original qubit are both $\frac{5}{6}$ (which proved to be the optimal fidelity of oneto-two qubits universal cloner) for arbitrary input pure states.

\section{UNIVERSAL CLONING}

The most important quality of symmetric universal cloning machines is how well we can do, i.e., the optimal fidelity. We use the most usual definition of fidelity $F=\langle\Psi|\rho| \Psi\rangle$. For convenience, we only discuss the case when the input states are pure. Here, $|\Psi\rangle$ is the to-becloned unknown pure state and $\rho$ is the density matrix of one of the output copies.

A $M \rightarrow N$ symmetric universal quantum cloning machine is described as the following (for qubit systems):

1. The input system contains $M$ original qubits, each in the same unknown pure state $|\Psi\rangle, N-M$ "blank" qubits $|0\rangle$ and $K$ auxiliary qubits $|0\rangle$.

2 . The cloning machine acts on the input system and performs a prescribed unitary transformation $U$ on it. 
3. The output system contains $N$ identical copies (usually entangled), each copy described by a reduced density matrix $\rho_{i}^{\text {out }}(i=1, \cdots, N)$, and $K$ auxiliary qubits.

The cloning process can be written as

$$
U|\Psi\rangle^{\otimes M}|0\rangle^{\otimes(N-M)}|a u x\rangle=|\Phi\rangle^{\text {out }} .
$$

For $|\Phi\rangle^{\text {out }}$, there exists $N$ qubits such that each of them has the same reduced density matrix $\rho^{\text {out }}$.

For the $M \rightarrow N$ symmetric universal quantum cloning machine described above, the optimal fidelity has been demonstrated as [10]

$$
F^{o p t}(M, N)=\frac{M N+N+M}{N(M+2)} .
$$

From the equation we can see that the optimal $1 \rightarrow 2$ fidelity is $\frac{5}{6}$.

The special unitary transformation $U$ of the one-to-two Bužek-Hillery cloning machine can be written as [4]

$$
\begin{gathered}
|0\rangle_{1}|0\rangle_{2}|0\rangle_{3} \stackrel{U}{\longrightarrow} \sqrt{\frac{2}{3}}|00\rangle_{12}|0\rangle_{3}+\sqrt{\frac{1}{3}}|+\rangle_{12}|1\rangle_{3}, \\
|1\rangle_{1}|0\rangle_{2}|0\rangle_{3} \stackrel{U}{\longrightarrow} \sqrt{\frac{2}{3}}|11\rangle_{12}|1\rangle_{3}+\sqrt{\frac{1}{3}}|+\rangle_{12}|0\rangle_{3},
\end{gathered}
$$

where

$$
|+\rangle=\sqrt{\frac{1}{2}}\left(|10\rangle_{12}+|01\rangle_{12}\right)
$$

In the cloning process, the quantum information in the original qubit (the first qubit) is copied to qubits 1 and 2 equally well such that the reduced density matrixes at the output are identical, i.e., $\rho_{1}^{\text {out }}=\rho_{2}^{\text {out }}$. The third qubit acts as an auxiliary qubit here.

From the specific expression of the unitary transformation $U$ given above we can easily calculate the fidelities

$$
\begin{aligned}
& F_{1}=\left\langle\Psi\left|\rho_{1}^{\text {out }}\right| \Psi\right\rangle=\frac{5}{6}, \\
& F_{2}=\left\langle\Psi\left|\rho_{2}^{\text {out }}\right| \Psi\right\rangle=\frac{5}{6},
\end{aligned}
$$

where $|\Psi\rangle$ is an arbitrary unknown pure state

$$
|\Psi\rangle=\alpha|0\rangle+\beta e^{i \varphi}|1\rangle, \alpha, \beta \in \text { real, } \varphi \in[0,2 \pi) .
$$

So, it is shown to be an optimal symmetric universal cloning machine.

In order to realize the transformation $U$ in experiment, we first have to construct the quantum logic circuit. To do that, $U$ is decomposed into a sequence of basic operations such as the rotation of a single qubit and the controlled-NOT (CNOT) operation of two qubits 22. The final network of the cloning machine [14] has been shown in figure 1.

We note that the network is divided into two parts (except the state swapping part): preparation and cloning. In the preparation stage, qubit 1 is left unaffected and qubit 2 and 3 are entangled by CNOT gates. That is, there is no "flow" of quantum information from qubit 1 to qubit 2,3 . Then, in the cloning stage, the quantum information in qubit 1 is "redistributed" among the three qubits by a sequence of four CNOT gates.

The unitary transformations in the two stages are described as 14

$$
|\Psi\rangle_{1}|0\rangle_{2}|0\rangle_{3} \stackrel{U_{1}}{\longrightarrow}|\Psi\rangle|\Psi\rangle_{23}^{\text {prep }} \stackrel{U_{2}}{\longrightarrow}|\Psi\rangle_{123}^{\text {out }},
$$

where $|\Psi\rangle_{23}^{\text {prep }}$ is prepared by three ratations and two CNOT operations

$$
|\Psi\rangle_{23}^{\text {prep }}=\hat{R}_{2}\left(\theta_{3}\right) \hat{P}_{32} \hat{R}_{3}\left(\theta_{2}\right) \hat{P}_{23} \hat{R}_{2}\left(\theta_{1}\right)|0\rangle_{2}|0\rangle_{3} .
$$

The rotation angles $\theta_{j}(j=1,2,3)$ are determined by the specific $|\Psi\rangle_{23}^{\text {prep }}$. In this case the state $|\Psi\rangle_{23}^{\text {prep }}$ reads

$$
|\Psi\rangle_{23}^{\text {prep }}=\sqrt{\frac{1}{6}}\left(2|00\rangle_{23}+|01\rangle_{23}+|11\rangle_{23}\right) .
$$

Although there is no flow of information in the preparation stage, we note that we can controll the distribution of information among the three qubits by the choice of the ratation angles [14]. We can copy qubit 1 to qubits 1 and 2 or qubits 2 and 3, even copy qubit 1 to qubits 1 , 2 and 3 when the input state $|\Psi\rangle$ is

$$
|\Psi\rangle=\alpha|0\rangle+\beta|1\rangle, \alpha, \beta \in \text { real }
$$

This is called quantum triplicator.

\section{EXPERIMENT DESCRIPTION}

Now we concentrate on constructing the network given in figure 1 with linear optical devices. The basic idea is the representation of several qubits by a single photon. In such a scheme, qubits are represented by different freedom degrees of a single photon, i.e., a photon can carry one polarization qubit and several location qubits [15 17]. Two orthogonal linear polarization states of the photon, i.e., horizontal and vertical, serve as the basis states of the polarization qubit. Hereafter, they are denoted by $|H\rangle$ and $|V\rangle$ corresponding to $|0\rangle$ and $|1\rangle$, respectively. Any of the location qubits is characterized by the information about "which path" is taken by the photon when passing a beam splitter. In this way, entanglement of different qubits can be accomplished conveniently, and decoherence is very low because the photons have relatively less interaction with the environment.

In our scheme of universal quantum cloning, three qubits are involved: one polarization (qubit 1) and two location qubits (qubits 2 and 3). Qubit 1 is the initial to-be-cloned qubit in an arbitrary state $|\Psi\rangle_{1}$, which is cloned to qubits 1 and 2 at the output end with qubit 3 serving as an ancilla. Both location qubits are initially in blank states: $|0\rangle_{2}$ and $|0\rangle_{3}$. 
To realize the network in figure 1, we must construct CNOT gates between any two qubits and unitary rotation of any single qubit. With the representation of qubits mentioned above, all these gates can be made with linear devices [16,18. However, in previous schemes [18], a unitary rotation to a single location qubit is realized by a shiftable beam splitter (BS) or a Mach-Zehnder (M-Z) interferometer. Unfortunately, a shiftable BS is infeasible and an M-Z interferometer will inevitably multiply the complexity.

To overcome this difficulty, a trick of state-swapping between qubits 1 and 2 is employed to transform the input state $|\Psi\rangle_{1}|0\rangle_{2}|0\rangle_{3}$ to $|H\rangle_{1}|\Psi\rangle_{2}|0\rangle_{3}$. Then with a definite polarization state, unitary rotations to a single location qubit can be realized by an operation of ControlledRotation (polarization controls location), which can be implemented by only using one Polarization Beam Splitter (PBS) and several properly oriented Half-Wavelength Plates (HWP).

After the state-swapping, we can begin to construct the two parts of our network: preparation and cloning. After having finished this work, we may have a complicated construction (composed of many universal logic gates) of such a cloning machine with optical deveces. But by techniques of compiling [17], the network can be significantly simplified and a feasible setup is shown in figure 1.

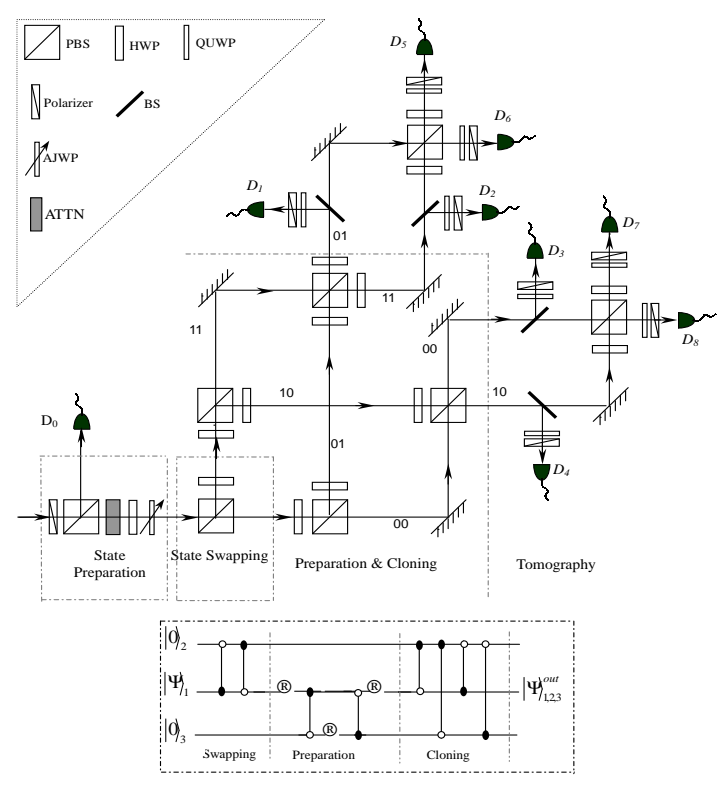

FIG. 1. the setup of the universal quantum cloning machine.

Now let's explain the experiment setup (figure 1) in detail.

The performance of this cloning machine is demonstrated by using a laser beam attenuated to the singlephoton level as input source. At the output, the photons are detected using geigermode avalanche photodiodes - Single Photon Counting Modules (D1-D8, EG\&G \#SPCM-AQ, efficiency $\sim 70 \%$, dark count $\sim 50 s^{-1}$ ). In our experiment, a well polarized light beam is created by a He-Ne laser with a Brewster window (Melles Griot, $633 \mathrm{~nm}$ ) and its polarization degree is improved by a polarizer. Then a PBS follows, and its vertical output is measured by a power meter $D_{0}$ to confirm the stability of the laser's power during the experiment. The horizontal output of this PBS is greatly attenuated (by an attenuating plate, ATTN) so that the maximum detection rates are always less than $20000 \mathrm{~s}^{-1}$; for the setup passage time of $5 \mathrm{~ns}$, this means that on average fewer than $10^{-4}$ photons are in the setup at any time. Now the input source can be viewed as single-photon source [19].

After that the to-be-cloned state is prepared by a properly oriented HWP and an adjustable waveplate (AJWP, namely, a Pockels Cell).

To confirm the correct result, in a general way, quantum state tomography [19] at the output end is required, and a simple version by optical interference is considered in the present system. Another auxiliary qubit is utilized to determine the reduced density operators of the two replicas respectively. This auxiliary qubit (qubit aux) is introduced as $\frac{1}{\sqrt{2}}\left(|0\rangle_{a u x}+|1\rangle_{a u x}\right)$ by four $50-50$ BSes. Then a controlled state swapping is performed with qubit aux as the controller: if qubit aux is $|1\rangle_{\text {aux }}$, the states of qubits 1 and 2 interchange, or else they remain unchanged. The four paths with qubit aux as $|0\rangle_{\text {aux }}$ determine the density operator of qubit 1 (polarization): if the relative photon counts and the polarization state of path $i$ is $C_{i}$ and $\rho_{i}$ respectively (by measuring four linearly independent states of the polarization qubit: $|H\rangle,|V\rangle,|D\rangle$, $|R\rangle$, we can determine each component of the matrix $\rho_{i}$, here $|D\rangle=\frac{1}{\sqrt{2}}(|0\rangle+|1\rangle),|R\rangle=\frac{1}{\sqrt{2}}\left(|0\rangle+e^{i \frac{\pi}{2}}|1\rangle\right)$, and this method is called quantum tomography [19]), the density operator of qubit 1 can be written as $\sum C_{i} \rho_{i}$. Other four paths determine the density operator of qubit 2 (location) which has been swapped to the polarization qubit with aux as $|1\rangle_{a u z}$, and the method of measurement is the same as above.

Then the reduced density operators of the replicas provide the evaluation of the fidelities, which are shown in Figure 2. We can see that the experiment datas coincide with the theoretical value $\frac{5}{6}$ quite well.

Before qubit 2 is under verification, it has been swapped with the polarization one, so errors of both qubits' fidelities can be analyzed in similar ways. Now we will analyze the error of our experiment. The error of the fidelity $F$ originates mainly from two ways:

1. The oscillation of the photon counts of pass $i$ (denoted by $\Delta C_{i}$ ), which is primarily caused by the small oscillation of the reflexivity and transmissivity of the first 
HWP (in the stage of state preparation) for different polarization states.

2. The precision limits of the waveplates and polarizers' orientation angles (denoted by $\Delta \theta$ ).

It can be deduced that an upper bound of the maximum error of $F$ can be expressed as 20] $\Delta F=\sum_{i=1}^{4} \Delta C_{i}+$ $\frac{3}{2} \Delta \theta$. In the present system, $\Delta \theta \approx 0.0018 \mathrm{rad}\left(0.1^{\circ}\right)$ and $\sum_{i=1}^{4} \Delta C_{i} \approx 0.002$. Thus we get the maximum error of $F$ as $\Delta F \approx 0.005$, which has been embodied in the result figure as error bars.

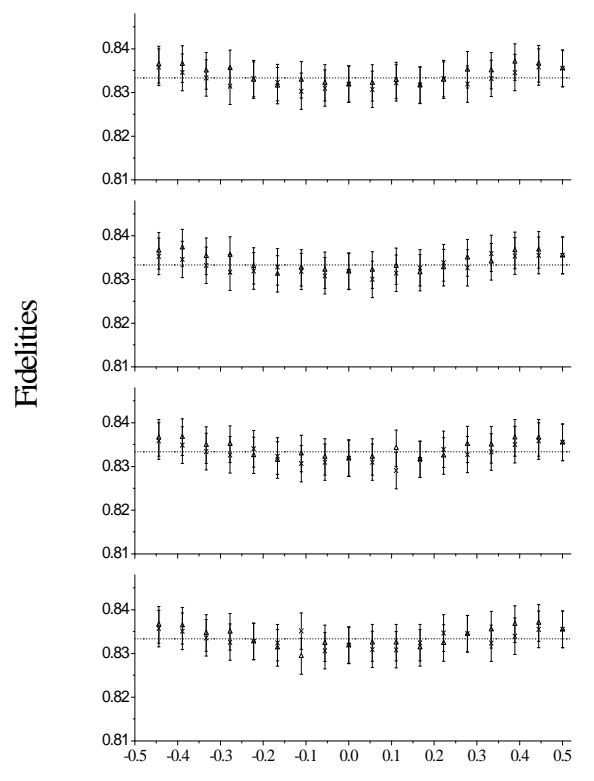

State Angle ( $\pi$ rad)

FIG. 2. the replicas' fidelities in the optimal universal cloning machine.

A more complicated network can be designed to carry out the $N \rightarrow M$ cloning, whose principle has been generalized from the $1 \rightarrow 2$ cloning [4, 10]. But in the present scheme, qubits are represented by different freedom degrees of a single photon, and about $2^{n}$ optical elements are needed to represent $n$ qubits [16]. So the complexity of the network will increase exponentially with the increase of $N$ and $M$, and this is a shortcoming of the present method.

In the present experiment, qubits are represented by different freedom degrees of a single photon. With a quantum non-demolition (QND) measurement [21], the information of the location qubit may be extracted without destroying the polarization qubit, in principle.

\section{SETUP AND RESULTS}

Figure 1 is the setup of the universal quantum cloning machine. The inset is the logic circuit $\bullet$ and $\circ$ denote the controller and target of a CNOT operation respectively, $R$ is a rotation to a single qubit). We represent the three input qubits by three freedom degrees of a single photon. Qubit 1 (to-be-cloned polarization qubit) is introduced by setting the photons' initial polarization in an arbitrary state $|\Psi(\theta, \delta)\rangle_{1}=\cos \theta|H\rangle_{1}+$ $e^{i \delta} \sin \theta|V\rangle_{1}\left(-\frac{\pi}{2}<\theta \leq \frac{\pi}{2}, 0 \leq \delta<\pi\right)$. Qubits 2 and 3 are both location qubits, which are introduced by different choices of "beam paths" with the initial state $|00\rangle_{2,3}$. By the state-swapping, qubit 2 is transformed to $|\Psi(\theta, \delta)\rangle_{2}=\cos \theta|0\rangle_{2}+e^{i \delta} \sin \theta|1\rangle_{2}$, while qubit 1 is transformed to $|H\rangle_{1}$. In the stage of entanglement preparation, qubits 1 and 3 are prepared into $|\Psi\rangle_{1,3}^{\text {prep }}=\frac{1}{\sqrt{6}}\left(2|H 0\rangle_{1,3}+|H 1\rangle_{1,3}+|V 1\rangle_{1,3}\right)$. Then several CNOT operations follow to accomplish the cloning, which can be compiled to two independent polarization M-Z interferometers. Quantum state tomography is adopted to confirm the correct result. Another auxiliary qubit enables us to measure the density operators of the replicas separately, which is introduced as $\frac{1}{\sqrt{2}}\left(|0\rangle_{\text {aux }}+|1\rangle_{\text {aux }}\right)$ by arranging four 50-50 BSes in each potential paths of the output photon. Then a controlled state swapping is carried out and the four qubits (qubit 1, 2, 3 and $a u x$ ) evolve to the state $|\Psi\rangle^{\text {meas }}=$ $\frac{1}{\sqrt{2}}\left(|0\rangle_{\text {aux }}|\Psi\rangle_{1,2,3}^{\text {out }}+|1\rangle_{\text {aux }}|\Psi\rangle_{2,1,3}^{\text {out }}\right)$. When the qubit aux is $|0\rangle_{\text {aux }}$, the density operator of replica qubit 1 (polarization) can be obtained by detectors 1 to $4\left(D_{1}-D_{4}\right)$ and corresponding QUWPs and polarizers, by measuring the mixed state of polarization and relative photon counts of each path. To the other four outputs of the BSes where the qubit aux is $|1\rangle_{\text {aux }}$, a state-swapping between qubits 1 and 2 is performed so that the density operator of replica qubit 2 is exactly realized by knowing that of the interchanged polarization qubit, which is measured by $D_{5}-D_{8}$ and corresponding QUWPs and polarizers.

Figure 2 is the replicas' fidelities in the optimal universal cloning machine. The fidelity is defined as $\langle\Psi(\theta, \delta)|\rho| \Psi(\theta, \delta)\rangle$ with $\rho$ denoting the density operator of a replica. This machine can deal with any state $|\Psi(\theta, \delta)\rangle=\cos \theta|V\rangle+e^{i \delta} \sin \theta|H\rangle$ equally well. From down to up, the four subfigures represent the cases $\delta=0, \frac{\pi}{4}, \frac{\pi}{2}, \frac{3 \pi}{4}$, respectively. In each subfigure, the state angle $\theta$ of the to-be-cloned qubit varies from $-\frac{\pi}{2}$ to $\frac{\pi}{2}$, the dotted lines represent the theoretical optimal fidelities $\frac{5}{6}$ $(\approx 0.833)$, while the crosses $(x)$ denote the fidelities of the output qubit 1 and the triangles $(\triangle)$ denote the fi- 
delities of qubit 2. As is shown, all the fidelities are close to the theoretical value $\frac{5}{6}$, and errors have been analyzed in section III.

\section{CONCLUSION}

We have realized a one-to-two qubits Bužek-Hillery cloning machine with linear optical devices. We showed that, the fidelities between the two output qubits and the original qubit are both $\frac{5}{6}$ for arbitrary input pure states.

Acknowledgment This work was supported by the National Natural Science Foundation of China.
[1] W. K. Wootters and W. H. Zurek, Nature (London) 299, 802 (1982).

[2] H. P. Yuen, Phys. Lett. A 113, 405 (1986); G. M. D'Ariano and H P.Yuen, Phys. Rev. Lett. 76, 2832 (1996).

[3] M. Koashi and N. Imoto, Phys. Rev. Lett. 81, 4264 (1998).

[4] V. Bužek and M. Hillery, Phys. Rev. A 54, 1844 (1996).

[5] L.-M. Duan and G.-C. Guo, Phys. Lett. A 243, 261 (1998); L.-M. Duan and G.-C. Guo, Phys. Rev. Lett. 80, 4999 (1998).

[6] A. Chefles and S. M. Barnett, Phys. Rev. A 60, 136 (1999).

[7] N. J. Cerf, J. Mod. Opt. 47, 187 (2000).

[8] V. Bužek, M. Hillery, and R. Rednik, Acta Phys. Slov. 48, 177 (1998).

[9] N. Gisin and S. Massar, Phys. Rev. Lett. 79, 2153 (1997); D. Bru $\beta$, D. P. DiVincezo, A. Ekert, C. A. Fuchs, C. Macchiavello, and J. A. Smolin, Phys. Rev. A 57, 2368 (1998).

[10] D. Bruss, A. Ekert, and C. Macchiavello, Phys. Rev. Lett. 81, 2598 (1998).

[11] E. F. Galvão and L. Hardy, quant-ph/0002053.

[12] N. Gisin, B. Huttner, Phys. Lett. A 228, 13 (1997).

[13] G. M. D'Ariano, C. Macchiavello and M. F. Sacchi, quant-ph/0007062.

[14] V. Bužek, S. L. Braunstein, M. Hillery\&D. Bru $\beta$, Phys. Rev. A 56, 3446 (1997).

[15] M. Zukowshi, Phys. Lett. A 157, 198 (1991).

[16] N. J. Cerf, C. Adami\& P. G. Kwiat, Phys. Rev. A 57, 1477 (1998).

[17] P. G. Kwiat, J. R. Mitchell, P. D. D. Schwindt\&A. G. White, J. Mod. Opt. 47, 257 (2000).

[18] A. Zeilinger, Am. J. Phys. 49, 882 (1981).

[19] A. G. White, D. F. V. James, P. H. Eberhard, and P. G. Kwiat, Phys. Rev. Lett. 83, 3103 (1999).

[20] Considering the output state of the polarization qubit as $\rho=\sum_{i=1}^{4} C_{i}\left|\Psi\left(\theta_{i}, \delta_{i}\right)\right\rangle\left\langle\Psi\left(\theta_{i}, \delta_{i}\right)\right|$, the fidelity is calculated by $F=\langle\Psi(\theta, \delta)|\rho| \Psi(\theta, \delta)\rangle$. Let $F=\sum_{i=1}^{4} f_{i}$, where $f_{i}=\frac{1}{2} C_{i}\left[1+\cos 2 \theta \cos 2 \theta_{i}+\sin 2 \theta \sin 2 \theta_{i} \cos \left(\delta_{i}-\delta\right)\right]$. It is easy to prove that $\Delta f_{i} \leq \Delta C_{i}+C_{i}\left(\Delta \theta_{i}+\frac{1}{2} \Delta \delta_{i}\right)$, and the error of $F$ can be expressed as $\Delta F=\sum_{i=1}^{4} \Delta f_{i}$. Then the maximum error may be written as $\Delta F=$ $\sum_{i=1}^{4} \Delta C_{i}+\frac{3}{2} \Delta \theta$, for $\sum_{i=1}^{4} C_{i}=1$ and $\Delta \theta_{i}=\Delta \delta_{i}=\Delta \theta$ is the orientation precision.

[21] G. Nogues, A. Rauschenbeutel, S. Osnaghi, M. Brune, J. M. Raimond, \& S. Haroche, Nature, 400, 239 (1999).

[22] A. Barenco, C. H. Bennett, R. Cleve, D. P. DiVincenzo, N. Margolus, P. Shor, T. Sleator, J. A. Smolin and H. Weinfurter, Phys. Rev. A 52, 3457 (1995). 